\title{
El proceso de diseño desde la génesis de los materiales
}

\section{Baima, Marina Andrea}

Resumen: Las nuevas dinámicas de generación de producción y difusión del conocimiento, y la interrelación con las plataformas de innovación aplicadas a los sistemas productivos locales, definen nuevos escenarios de innovación.

La profunda comprensión de las operatorias de la complejidad contemporánea amplia el espectro de acción del proceso proyectual.

La inclusión de la génesis de los materiales en el proceso proyectual redefine las posibles hipótesis de diseño y fomenta una dinámica de cocreación entre múltiples disciplinas.

Palabras clave: nuevos materiales diseño industrial - innovación abierta -

Cuadernos del Centro de Estudios de Diseño y Comunicación №70

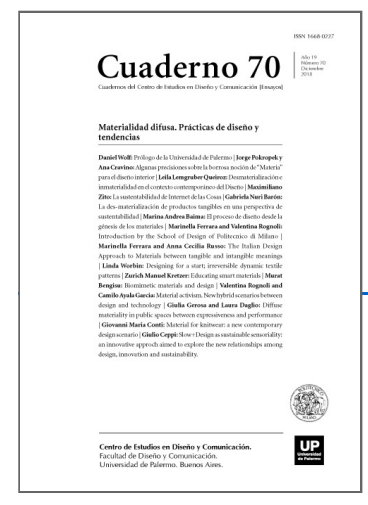

ISSN: 1668-0227

Materialidad difusa.

Prácticas de diseño y

tendencias

Año XIX, Diciembre 2018, Buenos Aires, Argentina | 172 páginas

descargar PDF ver índice de la publicación

Ver todos los libros de la publicación

compartir en Facebook

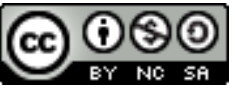
Esta obra está bajo una Licencia Creative Commons Atribución-NoComercialCompartirlgual 4.0 Internacional co construcción.

$\left(^{*}\right)$ Diseñadora Industrial, Posgrado en Gestión de Ciudades y Emprendimientos Creativos, maestrando en Ciencia Tecnología y Sociedad. Especializada en gestión estratégica CTI y dirección de proyectos centrados en las bioeconomías y el conocimiento colectivo.

Introducción

En el marco de un cambio de paradigma en las tecnologías y modos de producción, la generación de nuevos diagramas de producción del conocimiento, como la innovación abierta y la co-creación, surgen en un contexto en el cuál se le demanda a las industrias manufactureras una creciente diferenciación de sus productos.

Asimismo, la relevancia de las actividades de base tecnológica digital son cada vez más pronunciadas, y es creciente la vinculación de los productos a valores de sustentabilidad social, ecológica y cultural. Sobre la base de estas explotaciones, el diseño toma dimensiones cada vez más complejas. Este estudio pretende contribuir al análisis del diseño, como objeto de co-creación, a partir de un paquete de metodologías que orientan, ordenan y traducen procesos que favorecen la apertura de sus fronteras y promueven como resultado el desarrollo de nuevos materiales con aplicabilidad específica. 
En las últimas décadas se ha generado abundante literatura orientada a la indagación de las dimensiones analíticas que influyen y condicionan la construcción de la ciencia y tecnología en los procesos de nuevos materiales, aquí nos concentraremos en desarrollar un enfoque en el diseño y su proceso de innovación, como agente que contribuye en la apertura de la caja negra del proceso científico tecnológico para la generación de nuevos materiales; eficientes, sensibles, dinámicos y como resultado de creación consiente.

Sobre este objeto se intentará comprender e identificar dimensiones subyacentes de las actividades de diseño como proceso de intervención en la génesis de los materiales.

Proceso abierto creativo y bordes invisibles

La ciencia y la tecnología aparecen en las esferas de las discusiones actuales como moderadores o creadores de las soluciones que demandan las estructuras sociales dinámicas hacia el nuevo paradigma sostenible.

El diseño, dentro de éstos fenómenos de construcción de soluciones aplicables, replicables, apropiables, deben impulsar reflexiones de cómo la disciplina proyectual está mutando hacia un proceso de transformación transversal que surge del nexo entre ciencia, tecnología, cultura y poder. Es por ello, que no debe configurarse solo como un vestigio del formalismo y estética que muchas veces banaliza la actividad del diseño.

Entender que todo cambio paradigmático trae una fase de destrucción creadora (Schumpeter) para poner en vanguardia innovaciones radicales ayuda a comprender las nuevas vertientes del diseño que renace de sus últimas versiones devenidas del materialismo inmediato, para centrar y profundizar los nuevos conceptos en que la sociedad se encuentra demandando. Incorporar al proceso de diseño el entendimiento de la utilidad social, el centro de la necesidad real, permite reflexionar como a partir de metodologías proyectuales propias del diseño industrial, los materiales pueden incorporar estas lógicas sustentables y así obtener resultados innovadores y generadores de nuevas oportunidades.

En este contexto, los procesos de innovación proyectual, requieren nuevos mecanismos y herramientas híbridas de creación y gestión. Para el logro exitoso, las redes sociales de innovación abierta exigen nuevas estructuras y procesos; esfuerzos permanentes de moderación, coordinación y seguimiento para esta apertura y flexibilidad que pueden ser configurables en el desarrollo de nuevos materiales.

En las dimensiones de los productos industriales, la intensidad del cambio tecnológico, así como el incremento de la complejidad y la incertidumbre en los mercados a causa de un acortamiento en el ciclo de vida de los productos, del incremento en la movilidad de las personas y de la diseminación del conocimiento, han provocado que pocas empresas puedan innovar utilizando sólo sus recursos internos. En este aspecto, lo fundamental son los procesos que permiten generar e intercambiar el conocimiento dentro de las empresas (OECD, 2005). Comprender este proceso, sustenta la movilidad de las fronteras del diseño como estímulo creador para el proceso de innovación en la generatriz de los nuevos materiales.

Una parte central del diseño en los procesos de innovación se refiere a la forma de encontrar ideas que se puedan materializar y comercializar. En este contexto, el diseño como línea metodológica para la generación de materiales adaptables a las nuevas improntas del mercado, puede ser adoptado de manera estratégica para alcanzar y mantener innovaciones (Schumpeter, 1912, 1942; Rosenberg, 1982; Von Hippel, 1986). 
Conjuntamente con la estrategia de innovación, desde nuestro enfoque, el proceso debe configurarse de manera abierta (Chesbrough, 2003) en donde la innovación abierta se define como una estrategia para reorganizar, exteriorizar y mejorar la forma de innovar mediante alianzas de colaboración con otras organizaciones, las actividades de I+D+i, la gestión intelectualindustrial, la búsqueda de fuentes externas de conocimiento y los mecanismos alternativos de salida al mercado.

La génesis abierta de los nuevos materiales, desde este enfoque toma como partícipes la ciencia, la tecnología y al diseño, siendo el último una herramienta colaborativa para vehiculizar y dar soluciones viables a partir del conocimiento generado y aplicado hacia la génesis de los materiales como la unión transversal entre ciencia, como elemento creador, y sociedad como el soporte del lenguaje objetual.

La co-producción implica que, tanto en el orden natural como en el orden social, se produce conjuntamente, y que ninguno de los órdenes prevalece por encima del otro (Jasanoff, 2004), entendiendo a la dimensión del diseño como elemento sistémico que aporta a esa cadena de producción para la incorporación de lenguajes no clásicos hacia los materiales y su fabricación. La información aplicada no es solo constructiva, sino simbólica, interpretativa, de exploración y manipulación en torno a una funcionalidad ampliada y dirigida hacia el usuario. (Norman \& Draper, 1986)

El entorno que se propone pensar es el que subyace a la sociedad en un ecosistema formado a través de materiales, que se transformar y conforman en ese entorno a partir de las capacidades de evolución a productos de diseño industrial, siendo sus significados, pregnancias, utilidades y motivos contextuales los que definen ese entorno y recrean las relaciones entre los individuos y los objetos.

Desde el objeto de estudio de la génesis de los materiales, a través de tecnologías proyectuales de diseño, el ecosistema conformado como material, construido a través de dinámicas de innovación abierta, sectoriales, productivas y de desarrollo científico y tecnológico, deben validarse y aceptarse en el mercado.

Desde esta hipótesis, la secuencia proyectual y sus barreras de co-coonstrucción empiezan a dilatarse para encontrar un sentido más comprometido, que puede re orientar ese conocimiento científico abstracto, hacia algo tangible, amigable, electivo que de un pasaje hacia una utilización, apropiación, difusión y transmisión de ese conocimiento experto, hacia un material objeto como resultado de un proceso proyectual.

El diseño como paquete tecnológico para el desarrollo de un nuevo material, se inscribe en un proceso complejo que implica interdisciplinariedad para su factibilidad. El objeto de estos nuevos materiales como portador de utilidad social, devienen de una metodología de diseño proyectual como elemento que interpreta las necesidades de los nuevos usuarios, y se complementan con otros grupos heterogéneos de conocimientos para que el procesos innovativo recree estos materiales.

Es así, que el diseño industrial se identifica como un elemento partícipe de la co-construcción de los nuevos materiales, que proporciona el conocimiento del entendimiento de las nuevas necesidades y que se constituye como un proceso creativo, tecnológico y multidisciplinar, orientado a la creación como proceso de nuevos materiales con utilidad social.

Revalorizar los ciclos activos y el pensamiento crítico de la materialidad y vida del producto 
Las dimensiones sustentables son fenómenos contemporáneos que ponen temáticas en cambio climático, biodiversidad, ética y equidad para intentar resignificar la demanda cultural. Estas premisas permiten reflexionar cómo los sistemas materiales, relevantes por su papel protagónico al ser una unidad compositiva que configura productos y objetos para el desarrollo de la interface humana, deban re enfocarse frente a estas necesidades sociales y naturales con conciencia ecológica, humana y económica. Donde conocimiento metodológico del diseño puede desempeñar un propósito integrador que confluya en sistemas tecnológicos, científicos, biológicos, físicos con ciencias sociales, antropología, sociología, a través de su propuesta proyectual.

La ciencia y la tecnología definen nuevos escenarios que dan una perspectiva para las oportunidades en donde el diseño puede proponer aportes dentro de los procesos de creación colaborativa. La sociedad de la información y el conocimiento están cambiando los modos de entender el diseño y modificando los perfiles profesionales sociales, estratégicos y sustentables.

Los nuevos materiales toman dimensiones de compuestos biológicos y tecnológicos: biomateriales, nanomateriales, materiales imprimibles, materiales compuestos y los futuros pre configurables. Nacen como consecuencia de la convergencia de diversas ramas del conocimiento.

En este escenario, nos enfocaremos a los biomateriales, como potencial de desarrollo estratégico en Argentina, donde por las ventajas competitivas propias de nuestro país se pueden generar una nueva disciplina creativa emergente y donde los esfuerzos relevantes de I+D dan resultados prácticos, estratégicos, con interés social, económico y productivo. La nanotecnología permite desarrollar materiales configurables átomo por átomo, dando oportunidad al diseño en pensar esa configuración para realzar propiedades y pensar desde lo taxativo mediante un enfoque sistémico que contribuya a conceptualiza y evaluar las ideas para poder llevarlas a un formato funcional y formal traducido a un material o nuevas estructuras tecnológicas.

En el campo de la génesis de los materiales, el diseño como tecnología puede pensarse en la desconstrucción de las propiedades técnicas y mecánicas para que su desempeño permee a las demandas desde las dimensiones de: percepción, estética, emoción y significado, adoptando una actitud reflexiva sobre la relatividad del material como producto que emerge hacia un contexto complejo. Así el apoyo teórico/metodológico del diseño, pueda estructurar la actividad creadora para aumentar las capacidades y reflexiones sobre los materiales y su impacto.

Esta situación de innovación colaborativa es un reto hacia el diseño en donde el re ordenamiento molecular de la biología y la nanotecnología como motor de las nuevas oportunidades pragmáticas y sintácticas pensando el diseño desde materiales a partir de la reconfiguración de los conocimientos existentes o la recombinación de los mismos son el soporte de la dimensión del diseño de estructuras con amplias posibilidades y configuraciones que determinen las características de los materiales.

Como promotor de estas dinámicas, los Fab Labs o Laboratorios de Fabricación Digital se inscriben en un cambio de paradigma en las tecnologías y modos de producción, que ayudan a las dinámicas de los nuevos materiales que surgen en un contexto en el cuál se le demandan a las industrias manufactureras una creciente diferenciación de sus productos. Asimismo, la relevancia de las actividades de base tecnológica digital son cada vez más pronunciadas, y es creciente la vinculación de los productos a valores de sustentabilidad social, ecológica y cultural. 
En este marco, la revolución informática está dando lugar a una nueva revolución industrial, tal como lo desarrolla Chris Anderson en su libro Makers, The New Industrial Revolution. Estamos rodeados por bienes físicos y la mayoría de ellos son producto de una economía industrial que durante el siglo pasado se transformó de todas las maneras posibles siguiendo una fuerte production push. Es aquí donde las nuevas estrategias de generación de conocimiento comienzan a tener un rol central, en tanto posibilitan la fabricación innovadora de productos físicos, en ambientes que facilitan la colaboración abierta y la transferencia.

La impresión 3D como herramienta naturalizada en el proceso de prototipado rápido en los diseñadores ya toma dimensiones propias y puede coadyuvar al diseño de materiales o pensarse como una reprogramación para este evento creativo material. En línea con la fabricación digital, como un fenómeno radical y de disrupción innovativa desde la revolución industrial, los materiales y su coexistencia objetual toman la misma radicalidad.

Desde esta perspectiva los materiales son un ciclo proyectual y son el insumo de soluciones creativas tanto por su génesis como por su descomposición. Los biomateriales, o materiales biológicos, transformados en a partir de fuentes renovables configurables en: materiales biodegradables, biocompatibles, bioinertes, para aplicarse en todas los ecosistemas necesarios para la sociedad y los aspectos culturales como base para definir las competencias de los materiales y su atractivo sustentable.

Experiencias materiales, diseños aplicados, proyecciones argentinas

En el contexto de los cambios en las dinámicas de producción de los materiales, señalamos al diseño como factor clave para generar esa adaptabilidad, siendo una tecnología de proceso. El diseñador como un eslabón del sistema, responde a las dinámicas de proyección de soluciones coherentes y coexistentes a la génesis innovativa.

Los recursos naturales renovables están siendo protagonistas. Se están debatiendo el "cómo" se afronta ese cambio y "quiénes" serán los protagonistas de generar ese acercamiento a su utilización y apropiación constante. Sobre la base de lo expuesto anteriormente podemos decir que existen al menos tres planos que tienen especificidades en sus contenidos y diferentes aplicaciones: ciencia, tecnología y diseño.

La identificación de las problemáticas asociadas a los nuevos paradigmas de los consumidores recrean una visión aguas abajo de las cadenas proyectuales, donde el desarrollo de nuevos productos con los indicadores de eficiencia, sustentabilidad y el nuevo contexto paradigmático recrean y se reformulan a través de nuevos escenarios.

La dinámica sobre la cual se sustenta este estudio como punto de partida hacia las aplicaciones en el campo de la búsqueda de soluciones abiertas y diseñadas para los nuevos materiales, en donde la materia prima y su vida útil deben ser congruentes al tiempo, modo de uso de los productos y que su ciclo contemple su procesamiento, impacto ambiental. Desde el objeto de este estudio, avanzamos en la exploración de casos paradigmáticos locales donde existen aportes e integraciones de la disciplina del diseño en la génesis de nuevos materiales.

Placa biodegradable orgánica para la incorporación de nutrientes y celulosa de manera directa

El objeto de este caso visibiliza el material como consecuencia de su necesidad funcional, el desarrollo termina en fase producto: surcos autoportantes biodegradables para ser utilizado en el momento de siembra, cuya 
materialidad interpreta el ciclo de producto que es el mismo de su ciclo de vida.

Es un compuesto de material orgánico que incorpora nutrientes y celulosa y se aglutina aplicando calor y aglutinante para su conformación final. En contacto con el agua, comienza a desarmarse y se incorpora al suelo, al cual aporta sus diversos componentes y es el punto más innovativo del material. Bajo este funcionamiento, el método permite, evitar la erosión y la pérdida de nutrientes, sin modificar el sistema de regado por surco, pero haciéndolo biodegradable.

El material que queda depositado en la tierra luego de su utilización, pasa a ser parte de ella y la nutre para el próximo ciclo productivo. La placa puede aplicarse a surcos longitudinales y perpendiculares durante los distintos períodos de sembrado, tanto en verano como en invierno, para responder a las diferentes condiciones climáticas y a las necesidades de las hortalizas en los cinturones verdes de las ciudades.

El proyecto fue desarrollado por una técnica agropecuaria y estudiante de Diseño Industrial. Analía Rojas (Agencia CTyS).

Algatex. Textil dermo-protector con algas marinas

Textil con propiedades dermo-protectoras, desarrollado a partir de configuraciones nanotecnológicas para el aprovechamiento sustentable del recurso natural: algas marinas, que tienen un potencial en la incorporación de sustancias químicas relevantes en dermatología. El material textil está orientado al campo del cuidado de la piel. El desarrollo del proyecto está siendo llevado a cabo por D.I. Ma. Alejandra Martínez en el Instituto de Investigaciones en Ciencia y Tecnología de Materiales en el área de materiales compuestos (COMP) - INTEMA (CONICET-UNMdP), en una escala de laboratorio, para luego proyectarlo a una escala de producción industrial.

Ceras biodegradables a partir de aceite de soja

Ceras de soja a partir de procesos oleoquímicos con características orgánicas, biodegradables, no tóxicas ni nocivas para el medioambiente.

Estos productos tienen uso industrial y comercial, y alto valor de mercado. Específicamente para ser utilizadas como recubriendo de frutas y hortalizas frescas.

Es un bioagente de recubrimiento superficial para frutas y hortalizas. Tiene alta efectividad en abrillantar, proteger y minimizar la pérdida de peso.

A diferencia de las ceras existentes en el mercado nacional, éste es un producto orgánico que presenta una mayor performance y durabilidad que los productos sustitutos, es biodegradable y no tóxico, no es nocivo para el medioambiente y lo más destacado está en que no afecta a los consumidores finales.

Por sus características, puede competir a nivel internacional y a partir de la intervención del diseño estratégico se sometió a diferentes modificaciones moleculares para cambiar los puntos de fusión e incorporarse como antidesmoldantes, recubrimientos para embeberse en embalajes, entre otros. 
El propósito de estos ejemplos es avanzar en la definición de las capacidades requeridas para conseguir una penetración eficaz de las tecnologías, modelos proyectuales y productivos apropiados y apropiables en diferentes niveles del diseño de nuevos materiales, mediante el relevamiento de información y la construcción metodológica para la recolección de datos que permitan evaluar la situación actual y elaborar dinámicas para la génesis de los mismos. Se trata de romper el paradigma de los sistemas de producción lineales, hacia sistemas cí- clicos e interactivos. Así como los elementos naturales se transforman o son aprovechables por el mismo sistema, los productos también pueden ser pensados desde esta perspectiva metabólica (McDonough, 2002), trasladando este concepto al diseño de materiales.

Desde esta lógica, se visibiliza como el diseño puede ampliar sus posibilidades proyectuales a través de estrategias competitivas dentro de la cadena de valor de la génesis de los materiales, incorporando las dimensiones ambientales, sociales, productivos, sus cualidades físicas, entre otras oportunidades de nuevos productos y desempeños a partir de esta nueva concepción. El diseño industrial y los productos de su creatividad proyectual pueden enmarcase dentro de las nuevas configuraciones materiales, la introducción de cambios conceptuales de los materiales que los conforman hasta reconfiguraciones de nuevas sintaxis que devienen del re diseño, eco diseño, diseño competitivo y estratégico como co-esfera (Jassanof) de integración social, científica y tecnológica.

Podemos asociar como la historia ha sido marcada en su evolución a través de la significación del material en vínculo con su manipulación hacia el desarrollo, edad de piedra, edad de bronce y edad de hierro. En contraparte, podemos nombrar como la sociedad del conocimiento surge y como hoy ese conocimiento se acerca a las definiciones de apertura, donde la integración de la innovación, pilar en el diseño, como traductor de las necesidades y problemáticas hacia esa producción del material como símbolo de estas etapas de cambio de paradigma pueden construirse o reconstruirse desde la metodología proyectual. Las tecnologías avanzan como el mismo sistema científico, proyectual e industrial, desde el hierro como clave para las primeras revoluciones industriales hasta los polímeros y su secuencia de masividad, los biomateriales como producto de la ciencia en las barreras cercanas del conocimiento y la revolución biotecnológica y TIC. Pensando el rol del método proyectual del diseño puede limitar o extralimitar las posibilidades de configuraciones y oportunidades de desarrollo material.

Es el momento de evolucionar hacia la integración como elemento emergente de la construcción proyectual, donde el material como protagonista del ciclo portante del proceso de vida de producto, se inscribe en la red transversal del paquete tecnológico del diseño. Las necesidades actuales demarcan nuevas oportunidades entorno al desarrollo de materiales. El diseño en su actividad proyectual como un espacio de co-creación abierto, dinámico y participativo propone abordar la génesis de los materiales desde una óptica integrada social y científica hacia el nuevo paradigma de sostenibilidad.

\section{Bibliografía}

Ashby, M. (2005). Designing Hybrid Materials. In M. Ashby, Materials selection in Mechanicals Design (pp. 339376). Chambouleyron, M.; Arena, A. y Pattini, A. (..) Diseño de productos y desarrollo sustentable estrategias de 
revalorización de productos manufacturados para su introducción en un nuevo ciclo de vida. INCIHUSA (Instituto de Ciencias Humanas y Sociales) CRICYT-CONICET.

Chesbrough, H. (2003). Open Innovation: The New Imperative for Creating and Profiting from Technology. Harvard Business School.

Design Council (2002). "Competitive advantage through design”.

Hippel, E. (1986). Lead users: a source of novel product concepts.Volume 32 Issue 7, Pages 791-805.

Jasanoff, S. (2004). The Co-Production of Science and Social Order.

Galán, B. (2009). “El rol del diseño en las economías creativas”, en Impacto del Diseño en la Argentina, inti.

Karana, E.; Barati, B.; Rognoli, V. \& Zeeuw van der Laan, A. (2015). Material driven design (MDD): A method to design for material experiences. International Journal of Design, 9(2), 35-54.

Norman, D. A. \& Draper, S. W. (1986). User Centered System Design: New Perspectives on Human-computer Interaction. Maldonado, T. (1993). El diseño industrial reconsiderado. Barcelona: G. Gili.

Manzini, E. (1999). Strategic design, an introduction, conferencia dictada en el máster en Diseño estratégico del Politécnico de Milán.

McDonough, W. (2002). Design for the triple top line: new tools for sustainable commerce.

McDonough, W. (2007). Cradle-to-cradle design: creating healthy emissions - a strategy for eco-effective product and system design.

OCDE (1982a). Manual de Frascati, Medición de las Actividades Científicas y Tecnológicas.

Robert, V. y Yoguel, G. (2011). The complex dynamics of economic development, en Antonelli, C. (ed.), The system dynamics of technological change. Cheltenham: Edward Elgar.

Rosenberg, N. (1982). Inside the black box technology and economics. Cambridge, London.

Sanders, E. B.-N. \& Stappers, P. J. (2008). Co-creation and the new landscapes of design. CoDesign, 4(1), 5-18.

Sharifi, S. y Pawar, K. (1996). "Product design as a means of integrating differentiation", Technovation, vol. 16, $\mathrm{N}^{\circ} 5$.

Shigley, J. E. y Mischke, Ch. R. (1989). Diseño en ingeniería mecánica. McGraw-Hill.

Shumpeter, J. (1967). Teoría del desenvolvimiento económico. México: Fondo de Cultura Económica.

Tung, F.-W. (2012). Weaving with rush: Exploring craft-design collaborations in revitalizing a local craft. International Journal of Design, 6(3), 71-84. 
Yoguel, G.; Becerra, P. y Silva Failde, D. (2010). La caja negra del diseño: algunas consideraciones metodológicas aplicadas al sector de indumentaria en la Argentina. Redes, Diciembre-Sin mes, 75-112.

Walsh, V. (1995). Design, innovation and the boundaries of the firm, Research Policy, № 25.

Walsh, V. y Roy, B. (1985). "The designer as a gatekeeper in manufacturing industry”, Design Studies, № 6.

Walsh, V.; Roy, R. \& Bruce, M. (1988), “Competitive by design”, Journal of Marketing Management, N² 2.

Abstract:

The new dynamics of generation of production and diffusion of knowledge, and the interrelation with innovation platforms applied to the local production systems, define new innovation scenarios.

The deep understanding of the operations of contemporary complexity expands the spectrum of action of the design process.

The inclusion of the genesis of materials in the design process redefines the possible design hypotheses and encourages a dynamic of co-creation between multiple disciplines.

Key words: new materials - industrial design - open innovation - co-construction.

Resumo: As novas dinâmicas de geração de produção e difusão do conhecimento, e a inter-relação com as plataformas de inovação aplicadas aos sistemas produtivos locais, definem novos cenários de inovação.

A profunda compreensão das operatórias da complexidade contemporânea estende o espectro de ação do processo Projetual.

A inclusão da gênesis dos materiais no processo Projetual redefine as possíveis hipóteses de design e propicia uma dinâmica de co-criação entre múltiplas disciplinas.

Palavras chave: novos materiais - design industrial - inovação aberta - co-construção.

El proceso de diseño desde la génesis de los materiales fue publicado de la página 53 a página61 en Cuadernos del Centro de Estudios de Diseño y Comunicación №70 